\title{
Fundus fluorescein angiography and OCT
}

\author{
Deepak Gupta
}

Received: 12 June 2010 / Accepted: 12 July 2010 /Published online: 29 July 2010

(C) Springer-Verlag 2010

\section{Dear Editor,}

Gallego-Pinazo and associates [1] raise an interesting point, questioning to the continued need for fundus fluorescein angiography (FFA) in the current era of optical coherence tomography (OCT) for retinal vascular pathologies. They correctly point out that FFA is an invasive investigation associated with the potential for side-effects. However, it is also important to consider the pathology that is being investigated and the information that is required. Common indications are diabetic maculopathy, central and branch retinal vein occlusions

It must be remembered that FFA is a dynamic investigation that provides important information in addition to just the area of thickening. Any area of thickening will have a leakage point from which the fluid is tracking. It is important to identify the precise point(s) of leakage rather than just the total area that is thickened in the first instance. FFA is more likely to identify the source of leakage within a given area of thickening. This information can be provided in the early frames before the area of edema is filled with dye in a good quality FFA. This information is useful in allowing more precise targeting of leakage points, rather than performing a grid laser pattern and hoping to adequately treat critical leaking points. This is particularly important in instances where macular edema is encroaching upon the foveal avascular zone (FAZ). This also minimizes the laser energy that needs to be applied in this sensitive area, which has the potential for creating negative scotomas, and reducing colour vision and driving vision with serious repercussions on the patient's visual quality of life.

\section{Gupta $(\bowtie)$}

Ipswich General Hospital,

Heath Road,

Ipswich, UK

e-mail: dgupta_01@yahoo.com
Secondly, it is important to confirm that any clinically significant edema, particularly that which is causing reduced visual acuity, is perfused rather than ischemic. Confirmation of the latter would preclude treatment. This can only be confirmed by FFA. Thirdly, depending where the thickened area is located, it is useful to know the exact location and extent of the FAZ. Arguably, this information may be less relevant if the leakage is not clinically significant, as per ETDRS [2] definitions, and the leakage is significantly far from the perifoveal region.

Finally, as a practical consideration, it is useful to have a FFA picture available for reference when targeting the perifoveal area with laser. This provides a more detailed map, with vessels that can be used as reference points, that can allow more precise targeting of laser-treatmentrequiring areas in the fundus.

Bearing the above in mind, it seems sensible to utilise FFA in the context of treating common retinal vascular pathologies such as diabetic maculopathy and vein occlusions, where information such as the point of leakage, the location of the FAZ and presence and extent of ischemia are all critical information in planning and executing treatment. However, OCT remains an important additional tool in managing retinal vascular pathologies, with an indispensible role in confirming the clinical suspicion of macular pathology and monitoring response treatment.

\section{References}

1. Gallego-Pinazo R, Dìa-Llopis M, Marco-Dolz R, Martínez-Castillo S (2010) Guidance of macular laser photocoagulation: is fluorescine angiography still needed? Graefes Arch Clin Exp Ophthalmol 248:1047-1048

2. Early Treatment Diabetic Retinopathy Study Research Group (1985) Photocoagulation for diabetic macular edema. Early treatment diabetic retinopathy study report number 1 . Arch Ophthalmol 103(12):1796-1806 